\title{
8 Capital allocation principles as compositional data
}

In Chapter 6 it was shown that given an (absolute) capital allocation principle $\vec{K}$ with $K=\sum_{j=1}^{n} K_{j}$, its relative counterpart is defined as $\vec{x}$, where components are $x_{i}=K_{i} / K$. This chapter is devoted to show that relative capital allocation principles can be understood as belonging to the (standard) simplex. Following a nomenclature often used by geologists, any vector of the simplex is called a composition and any set of vectors in the simplex is called compositional data. This chapter first presents the metric space structure of the simplex. Secondly, it is shown that it is possible to move forward and backwards from relative capital allocation principles to compositions and the opposite. Applications of this relationship are illustrated with the data set used all along this book. This chapter is based on the study that we carried out in Belles-Sampera et al. [2016a].

\subsection{The simplex and its vectorial and metric structure}

Let us define the (standard) simplex $\mathscr{S}^{n}=\left\{\vec{z} \in \mathbb{R}^{n} \mid z_{j} \geqslant 0, j=1, \ldots, n\right.$, $\left.\sum_{j=1}^{n} z_{j}=1\right\}$ provided with a particular structure of vector and metric space. Any vector $\vec{z} \in \mathscr{S}^{n}$ is a composition and a set of vectors is called compositional data. We need to define the vector space and to enrich it later with a distance in order to be allowed to talk about this vector space as a metric space. Any set of vectors needs two operations (often called vector addition and scalar multiplication) in order to be called a vector space over $\mathbb{R}$. These operations must satisfy a set of particular properties. The vector addition must be commutative, associative, and a neutral element is needed. Moreover, for each vector, its additive inverse must exist. A scalar multiplication for a vector space over $\mathbb{R}$ combines a real number with a vector and, whatever this combination is, it is necessary that the combination must be- 
long again to the set of vectors. Additionally, a neutral element for the scalar multiplication must exist, and the distributivity of the scalar multiplication with respect to the vector addition and, on the other side, the distributivity of the vector addition with respect to the scalar multiplication must be both satisfied.

Following the notation provided in Aitchison and Egozcue [2005], a vector addition called perturbation (denoted by $\oplus$ ) and a scalar multiplication called powering (denoted by $\odot$ ) may be attached to the set $\mathscr{S}^{n}$. These operations are defined by expressions (8.1) and (8.2), respectively, where $\vec{x}, \vec{y} \in$ $\mathscr{S}^{n}$ and $\lambda \in \mathbb{R}$ :

$$
\begin{aligned}
& \vec{x} \oplus \vec{y}=\left(\frac{x_{1} \cdot y_{1}}{\sum_{j=1}^{n} x_{j} \cdot y_{j}}, \ldots, \frac{x_{n} \cdot y_{n}}{\sum_{j=1}^{n} x_{j} \cdot y_{j}}\right), \\
& \lambda \odot \vec{x}=\left(\frac{x_{1}^{\lambda}}{\sum_{j=1}^{n} x_{j}^{\lambda}}, \ldots, \frac{x_{n}^{\lambda}}{\sum_{j=1}^{n} x_{j}^{\lambda}}\right) .
\end{aligned}
$$

It can be proved that the simplex $\mathscr{S}^{n}$ provided with operations $\oplus$ and $\odot$ has a linear vector space structure of dimension $n-1$. An important function in the context of compositional data is the closure function, $\mathscr{C}$. The closure function applied to a vector in $\mathbb{R}^{n}$ returns another vector whose components are the components of the original vector divided by the sum of all the components of the original vector. Keeping this in mind, the following expressions hold:

$$
\vec{x} \oplus \vec{y}=\mathscr{C}\left[\left(x_{1} \cdot y_{1}, \ldots, x_{n} \cdot y_{n}\right)\right], \lambda \odot \vec{x}=\mathscr{C}\left[\left(x_{1}^{\lambda}, \ldots, x_{n}^{\lambda}\right)\right] .
$$

Moreover, assuming the vector space structure of $\left(\mathscr{S}^{n}, \oplus, \odot\right)$, the neutral element $\overrightarrow{0}$ of $\oplus$ can be deduced. Given a vector $\vec{x}$ such that $x_{i}>0$ for all $i$, the relationship $\vec{x} \oplus \vec{r}=\overrightarrow{0}$ informs that $\vec{r}$ is the inverse of $\vec{x}$ with respect to the perturbation operation, so it should be written as $\vec{r}=(-1) \odot \vec{x}$. In other words,

$$
\vec{r}=\left(\frac{1 / x_{1}}{\sum_{j=1}^{n}\left(1 / x_{j}\right)}, \ldots, \frac{1 / x_{n}}{\sum_{j=1}^{n}\left(1 / x_{j}\right)}\right) .
$$

Then, using this last expression and (8.1),

$$
\overrightarrow{0}=\vec{x} \oplus \vec{r}=\mathscr{C}\left[\left(\frac{1}{\sum_{j=1}^{n}\left(1 / x_{j}\right)}, \ldots, \frac{1}{\sum_{j=1}^{n}\left(1 / x_{j}\right)}\right)\right]=\left(\frac{1}{n}, \ldots, \frac{1}{n}\right),
$$

so the neutral element $\overrightarrow{0}$ of the perturbation operation is the composition with all of its $n$ elements equal to $1 / n$. 
Figure 8.1 Example of perturbation (addition) and powering (scalar multiplication) in $\mathscr{S}^{2}$

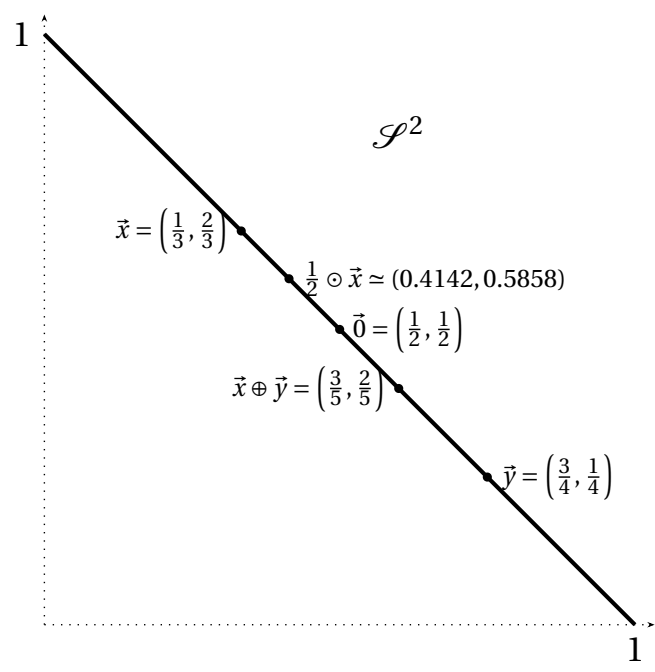

Example 8.1 (Perturbation, powering and neutral element in $\mathscr{S}^{\mathbf{2}}$ ). We provide an example in a low dimension $(n=2)$ in order to illustrate how the vector spaces $\left(\mathscr{S}^{n}, \oplus, \odot\right)$ work. Consider $\vec{x}=(1 / 3,2 / 3)$ and $\vec{y}=(3 / 4,1 / 4)$ in $\mathscr{S}^{2}$, and $\lambda=1 / 2 \in \mathbb{R}$. We can ask ourselves for $\vec{x} \oplus \vec{y}, \lambda \odot \vec{x}$ and $\overrightarrow{0}$

$$
\begin{aligned}
& \vec{x} \oplus \vec{y}=\left(\frac{1}{4} \frac{5}{12}, \frac{1}{6} \frac{5}{12}\right)=\left(\frac{3}{5}, \frac{2}{5}\right), \\
& \lambda \odot \vec{x}=\left(\frac{\sqrt{1 / 3}}{\sqrt{1 / 3}+\sqrt{2 / 3}}, \frac{\sqrt{2 / 3}}{\sqrt{1 / 3}+\sqrt{2 / 3}}\right) \simeq(0.4142,0.5858), \text { and } \\
& \overrightarrow{0}=\left(\frac{1}{2}, \frac{1}{2}\right) .
\end{aligned}
$$

For instance, $\vec{x} \oplus \overrightarrow{0}=\left(\frac{1}{6} \frac{1}{2}, \frac{1}{3} \frac{1}{2}\right)=\left(\frac{1}{3}, \frac{2}{3}\right)=\vec{x}$.

All these vectors are displayed in Figure 8.1.

Finally, a distance is needed in order to consider the vector space $\left(\mathscr{S}^{n}, \oplus, \odot\right)$ as a metric space. The simplicial metric defined in Aitchison [1983] is here considered. Given two compositions $\vec{x}, \vec{y}$, the distance between them from the point of view of the simplicial metric is

$$
\Delta(\vec{x}, \vec{y})=\left[\sum_{i=1}^{n}\left[\ln \left(\frac{x_{i}}{\operatorname{GM}(\vec{x})}\right)-\ln \left(\frac{y_{i}}{\operatorname{GM}(\vec{y})}\right)\right]^{2}\right]^{1 / 2},
$$


where $\operatorname{GM}(\vec{z})$ denotes the geometric mean of the components of $\vec{z}$ vector, this is $\operatorname{GM}(\vec{z})=\left[\prod_{i=1}^{n} z_{i}\right]^{1 / n}$.

An equivalent expression for $\Delta(\vec{x}, \vec{y})$ is the following:

$$
\Delta(\vec{x}, \vec{y})=\left[\frac{1}{2 n} \sum_{i=1}^{n} \sum_{j=1}^{n}\left[\ln \left(\frac{x_{i}}{x_{j}}\right)-\ln \left(\frac{y_{i}}{y_{j}}\right)\right]^{2}\right]^{1 / 2} .
$$

This simplicial metric is linked to a norm $\|\cdot\|_{\Delta}$ and to an inner product $\langle,\rangle_{\Delta}$ in a usual way. Given two vectors $\vec{x}, \vec{y} \in \mathscr{S}^{n}$,

$$
\Delta(\vec{x}, \vec{y})=\|\vec{x} \ominus \vec{y}\|_{\Delta}=\sqrt{\langle\vec{x} \ominus \vec{y}, \vec{x} \ominus \vec{y}\rangle_{\Delta}},
$$

where $\vec{x} \ominus \vec{y}=\vec{x} \oplus[(-1) \odot \vec{y}]$, and

$$
\langle\vec{u}, \vec{v}\rangle_{\Delta}=\frac{1}{2 n} \sum_{i=1}^{n} \sum_{j=1}^{n}\left[\ln \left(\frac{u_{i}}{u_{j}}\right) \cdot \ln \left(\frac{v_{i}}{v_{j}}\right)\right]
$$

Example 8.2 (Level curves in $\mathscr{S}^{\mathbf{3}}$ ). Once the distances are defined, we can explore - for instance - the geometrical locus of all those elements in the simplex with the same distance to a given element in that simplex. In other words, we could be interested in determining a sort of level curves in $\mathscr{S}^{n}$ related to the distances of compositions $\vec{x} \in \mathscr{S}^{n}$ to a fixed composition $\vec{y}_{0}$. Each one of these level curves would be driven by a certain $d \in \mathbb{R}^{+}$, the target distance. Formally, we could look for geometrical loci denoted $\operatorname{lc}_{d}\left(\vec{y}_{0}\right)$ and defined by

$$
\operatorname{lc}_{d}\left(\vec{y}_{0}\right)=\left\{\vec{x} \in \mathscr{S}^{n} \mid \Delta\left(\vec{x}, \vec{y}_{0}\right)=d\right\} .
$$

In Figure 8.2 several level curves are represented in a two dimensional projection of $\mathscr{S}^{3}$. On the left, $\mathrm{lc}_{d}(\overrightarrow{0})$ for $d=0.2,0.45,0.8$ and 1.0 are represented. As it is observed, the higher the distance to the composition of reference (in this case, $\left.\vec{y}_{0}=\overrightarrow{0}=(1 / 3,1 / 3,1 / 3)\right)$ the sharper the curve. Differences with respect to the Euclidean distance are evident, because these level curves have not circular shapes with center in the composition of reference. The behavior is similar on the right hand side of Figure 8.2. In that case, the composition of reference is $\vec{y}_{0}=(1 / 8,1 / 2,3 / 8)$ instead of the neutral element with respect to the perturbation (addition). The corresponding level curves $\operatorname{lc}_{d}\left(\vec{y}_{0}\right)$ for $d=0.2,0.45,0.8$ and 1.0 are once again represented but with this alternative reference. 
Figure 8.2 Examples of level curves $\operatorname{lc}_{d}\left(\vec{y}_{0}\right)$ in $\mathscr{S}^{3}$ are displayed. In fact, they are projections in $\mathbb{R}^{2}$ of the geometrical loci lc $\boldsymbol{d}_{d}\left(\vec{y}_{0}\right)$ of the elements which distances to point $\vec{y}_{0}$ are equal to $d$, where $d=0.2, d=0.45, d=0.8$ and $d=1.0$.

Left figure: $\vec{y}_{0}=(1 / 3,1 / 3,1 / 3)$ (neutral element with respect to the perturbation). Right figure: $\vec{y}_{0}=(1 / 8,1 / 2,3 / 8)$.
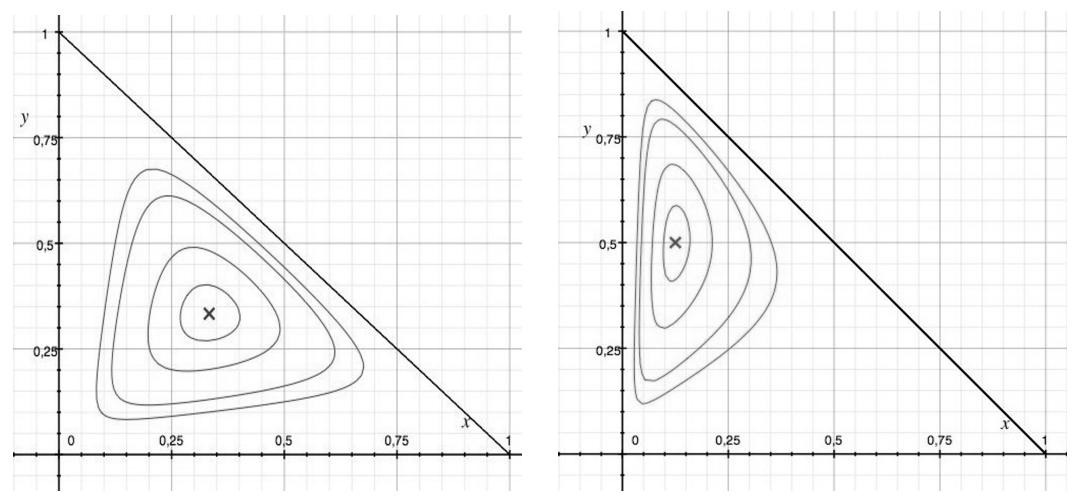

Under this framework, as it was shown in De Baets [2013], the simplicial arithmetic mean of the compositional data $\vec{x}_{1}, \vec{x}_{2}, \ldots, \vec{x}_{m}$ may be understood as a solution of a minimization problem, in the following way:

$$
\operatorname{AM}_{\Delta}\left(\vec{x}_{1}, \ldots, \vec{x}_{m}\right)=\frac{1}{m} \odot \bigoplus_{k=1}^{m} \vec{x}_{k}=\underset{\vec{z}}{\arg \min } \sum_{k=1}^{m}\left\|\vec{z} \ominus \vec{x}_{k}\right\|_{\Delta}^{2},
$$

where $\bigoplus_{k=1}^{m} \vec{x}_{k}$ means the perturbation of the set of $m$ compositions $\left\{\vec{x}_{k}\right\}_{k=1, \ldots, m}$. At first sight, this expression is equivalent to the arithmetic mean of $m$ real numbers $u_{1}, u_{2}, \ldots, u_{m}$ :

$$
\operatorname{AM}\left(u_{1}, \ldots, u_{m}\right)=\frac{1}{m} \cdot \sum_{k=1}^{m} u_{k}=\underset{v}{\arg \min } \sum_{k=1}^{m}\left\|v-u_{k}\right\|_{2}^{2},
$$

so, the simplicial metric presented in this section is the natural metric choice if (simplicial) arithmetic means are computed. In other words, the expression (8.6) contains the proper definition of the arithmetic mean of $\vec{x}_{1}, \ldots, \vec{x}_{m}$ in the metric space $\left(\mathscr{S}^{n}, \oplus, \odot, \Delta\right)$. From the definitions of both perturbation and powering operations, an explicit expression for the simplicial arithmetic mean presented in (8.6) is

$$
\mathrm{AM}_{\Delta}\left(\vec{x}_{1}, \ldots, \vec{x}_{m}\right)=\mathscr{C}\left[\left(G_{1}, \ldots, G_{n}\right)\right],
$$

where $G_{k}=G M\left(x_{1, k}, x_{2, k}, \ldots, x_{m, k}\right)$, i.e. $G_{k}=\left[\prod_{i=1}^{m} x_{i, k}\right]^{1 / m}$, for all $k=$ $1, \ldots, n$. 


\subsubsection{From capital allocation principles to compositional data}

An absolute capital allocation $\vec{K}$ has its relative counterpart $\vec{x}$ computed as $x_{i}=K_{i} / K$ for all $i=1, \ldots, n$. Note that it is satisfied that $\sum_{j=1}^{n} x_{j}=1$. Note also that when negative allocated capital amounts $K_{i}$ are allowed, the relative components would be negative and then $\vec{x} \notin \mathscr{S}^{n}$. For the rest of the chapter it is assumed that $\vec{x}$ has strictly positive components. That is, we assume that $\vec{x}$ is a composition with non-zero and non-negative components. This assumption allows to avoid negative or zero values on components of $\vec{x}$, which are an inconvenient for practitioners (negative allocations) and when operating in the simplex (null compositions) ${ }^{1}$.

At this point, some concepts introduced in Chapter 6 to classify absolute capital allocation problems can be associated to concepts introduced in this chapter. For instance, if we consider proportional capital allocation principles as stated in expression (6.1), the relative counterpart $\vec{y}$ of the absolute principle $\vec{K}=\left(K_{1}, \ldots, K_{n}\right)$ may be interpreted as the closure of the vector with components equal to $f_{i}\left(X_{i}\right), i=1, \ldots, n$ :

$$
\begin{gathered}
\vec{K} \text { s.t. } K_{i}=K \cdot \frac{f_{i}\left(X_{i}\right)}{\sum_{j=1}^{n} f_{j}\left(X_{j}\right)}, \forall i=1, \ldots, n \\
\Leftrightarrow \vec{y}=\mathscr{C}\left[\left(f_{1}\left(X_{1}\right), \ldots, f_{n}\left(X_{n}\right)\right)\right] .
\end{gathered}
$$

When stand-alone proportional principles are considered, the previous expression helps to visualize why dependence structures between random variables $\left\{X_{i}\right\}_{i=1, \ldots, n}$ are not taken at all into account in the capital allocation solution. In a first step, the amount of risk faced by each agent is assigned to one of them, which is summarized by $f_{i}\left(X_{i}\right), i=1, \ldots, n$. Subsequently, the relative risk proportion obtained in that manner is scaled by $K$ to obtain the final capital allocation.

\subsection{Perturbation inverse, simplicial distance and simplicial arithmetic mean applied to capital allocation problems}

In the previous section, it has been shown that relative capital allocation principles and compositions may be naturally linked. Once this relationship

1 Although elements of a composition can be equal to zero, dealing with compositions with null components is not an easy task in practice. 
is established, the idea is to take advantage of the geometric structure of the simplex to enrich the description of each capital allocation principle and each capital allocation result. Some applications of compositional methods in the context of capital allocation problems are shown in this section.

\subsubsection{The inverse of a capital allocation}

Let us consider a relative capital allocation principle $\vec{x}$ linked to what we have called in Chapter 6 a cost of risk goal. A manager would want to depart from this allocation to distribute rewards instead of costs in order to fulfill an allocation with a reward to an objective linked to minimisation of risk. An intuitive idea is to invert each of the relative components, in order to reflect the inverse nature of the allocation (a relative low cost allocated to $i$ th agent should mean a relative high reward assigned to him). To proceed in this direction, one must normalize the sum of all $1 / x_{i}$ in order to provide a full allocation of the whole amount of capital, $K$. Note that the inversion of the components is only feasible if all components of $\vec{x}$ are different from 0 . This application has a natural interpretation in the simplex $\mathscr{S}^{n}$. The normalization can be understood as the application of the closure function. Given a relative capital allocation principle $\vec{x}$, let $\vec{r}$ be the closure of the vector with components $1 / x_{i}$ for $i=1, \ldots, n$. As it has been shown in Section 8.1, $\vec{r}$ is the inverse of $\vec{x}$ with respect to the perturbation operation: $\vec{r}=(-1) \odot \vec{x}$.

Using risk based capital allocation principles to determine penalizations or rewards may lead to undesirable behaviors of the agents. Basically, agents have incentives to take conservative business decisions because less risk results in a better reward. In order to prevent it, a return-on-risk measure seems to be preferable to assign rewards. It has been indicated in Chapter 6 that rewards on risk and return allocations may be of great relevance for a sound ERM system.

Note now that there are some direct absolute reward on risk and return capital allocation principles that can be considered. For instance, if we depart from a given $\vec{x}=\mathscr{C}[\vec{y}]$, where $y_{i}=\operatorname{RORAC}\left(X_{i} \mid S\right) / \operatorname{RORAC}(S)$, for all $i=1, \ldots, n$.

Then, we obtain the absolute capital allocation principle $\vec{K}$ by

$$
K_{i}=K \cdot x_{i}=K \cdot \frac{\operatorname{RORAC}\left(X_{i} \mid S\right)}{\sum_{j=1}^{n} \operatorname{RORAC}\left(X_{j} \mid S\right)}, \forall i=1, \ldots, n .
$$

The underlying idea is to give a higher reward to those agents whose relative RORAC with respect to the overall RORAC of the portfolio is higher. 
Note that different definitions of return-on-risk measures than expressions (6.3) and (6.2) in Chapter 6 for $\operatorname{RORAC}\left(X_{i} \mid S\right)$ and $\operatorname{RORAC}(S)$ may be considered, and the objective of the allocation would not change.

\subsubsection{Ranking capital allocation principles}

We have presented a simplicial metric or distance $\Delta$ which helps to constitute $\mathscr{S}^{n}$ as a metric space. $\Delta$ can be used to quantitatively rank capital allocation principles. Let us consider the neutral composition $\overrightarrow{0} \in \mathscr{S}^{n}$ which is the composition with all of its $n$ components equal to $1 / n$. So, the distance between any relative capital allocation principle $\vec{x}$ and $\overrightarrow{0}$ can be computed. Alternatively, the distance between any pair of relative capital allocation principles belonging to $\mathscr{S}^{n}$ can be calculated. Both uses of the simplicial distance are useful to compare different capital allocation principles in a quantitative manner.

When the distance between the relative capital allocation $\vec{x}$ and $\overrightarrow{0}$ is computed, a quantitative result shows how far the allocation principle is from a neutral assignment. Note that $\overrightarrow{0} \in \mathscr{S}^{n}$ is linked to a capital allocation principle in which no matter how much risk each agent faces, they would all receive the same since the same amount is allocated to each one $(K / n)$. On the other hand, if an allocation principle is taken as a reference (for instance, a gradient allocation principle as explained in Section 6.3.1 of Chapter 6), the distance between the composition linked to this principle and any other composition quantifies how far this principle is from the allocation of reference.

Imagine that four allocation principles are in hand for the same amount $K$ of money and the same $n$ agents: a haircut allocation principle (7.2), $\vec{K}_{h}$; a covariance allocation principle $(6.15), \vec{K}_{c}$; a stand-alone proportional allocation principle based on GlueVaR $(7.5), \vec{K}_{s}$; and a gradient allocation principle related to (6.5), $\vec{K}_{g}$. If their respective relative allocation principles $\vec{x}_{h}, \vec{x}_{c}, \vec{x}_{s}$ and $\vec{x}_{g}$ are in $\mathscr{S}^{n}$ and each of the components of $\vec{x}_{t}, t \in\{h, c, s, g\}$ is strictly positive, then it is possible to rank them in two different ways:

1) Compute $\Delta\left(\vec{x}_{t}, \overrightarrow{0}\right)$ for $t \in\{h, c, s, g\}$. Order distances in an increasing order. A higher order position indicates an allocation located further apart from the neutral allocation;

2) Choose one of the principles as reference (for instance, the gradient allocation principle). Compute $\Delta\left(\vec{x}_{t}, \vec{x}_{g}\right)$ for $t \in\{h, c, s\}$. These three values are quantifying how far each principle is from the allocation of reference. 


\subsubsection{Averaging capital allocation principles}

In practice, different management teams may suggest different capital allocations regarding the same assignment problem. The situation sketched at the end of the previous section could be an example of such a situation. In those cases, we want to stress that the set of different capital allocation principles can be aggregated through the simplicial arithmetic mean, obtaining a final allocation that considers each one of the available viewpoints.

Formally, let us imagine $m$ management teams providing $m$ absolute capital allocation principles $\vec{K}_{k}$ of amount $K$ to the same $n$ agents, and let $\vec{x}_{k}$ be the relative capital allocation principles linked to $\vec{K}_{k}, k=1, \ldots, m$. Once again, taking advantage of the geometric structure of $\mathscr{S}^{n}$, the concept of averaging the $m$ points of view on the same allocation problem is easily derived. In other words, the expression $\vec{z}=\mathrm{AM}_{\Delta}\left(\vec{x}_{1}, \ldots, \vec{x}_{m}\right)$ is the proper definition of the arithmetic mean of $\vec{x}_{1}, \ldots, \vec{x}_{m}$ in the metric space $\left(\mathscr{S}^{n}, \oplus, \odot, \Delta\right)$ as it was shown in (8.6). Once the relative arithmetic mean is obtained, what remains to do is to assign an amount of $\bar{K}_{i}=K \cdot z_{i}$ monetary units to each $i$ th agent, $i=1, \ldots, n$, in order to provide a capital allocation principle in the adequate scale. This principle balances the opinions of all the involved management teams.

\subsubsection{An illustration}

In order to illustrate the applications described in this section we are getting back to the relative principles obtained in Chapter 7 , which where displayed in Table 7.2. Recall that these relative principles were derived from six absolute proportional allocation principles (6.1) based on three different GlueVaR risk measures but with two different perspectives: on the one hand, stand-alone proportional allocation principles (7.5) and, on the other hand, partial contributions based proportional allocation principles (7.9). Let us name them as $\vec{x}_{i}, i=1, \ldots, 6$. Then

$$
\begin{aligned}
\vec{x}_{1} & =(50.41 \%, 45.80 \%, 3.79 \%), \\
\vec{x}_{2} & =(63.51 \%, 28.38 \%, 8.11 \%), \\
\vec{x}_{3} & =(54.44 \%, 32.22 \%, 12.22 \%), \\
\vec{x}_{4} & =(46.42 \%, 51.74 \%, 1.84 \%), \\
\vec{x}_{5} & =(68.19 \%, 26.86 \%, 4.95 \%), \\
\vec{x}_{6} & =(25.11 \%, 73.11 \%, 1.78 \%) .
\end{aligned}
$$


Table 8.1 Perturbation inverse relative capital allocation principles.

\begin{tabular}{lccr}
\hline & $X_{1}$ & $X_{2}$ & $X_{3}$ \\
\hline & \multicolumn{3}{c}{ Stand-alone } \\
$\vec{y}_{1}=(-1) \odot \vec{x}_{1}$ & $6.50 \%$ & $7.15 \%$ & $86.35 \%$ \\
$\vec{y}_{2}=(-1) \odot \vec{x}_{2}$ & $9.03 \%$ & $20.22 \%$ & $70.75 \%$ \\
$\vec{y}_{3}=(-1) \odot \vec{x}_{3}$ & $14.00 \%$ & $23.65 \%$ & $62.35 \%$ \\
& & & \\
$\vec{y}_{4}=(-1) \odot \vec{x}_{4}$ & $3.68 \%$ & $3.30 \%$ & $93.02 \%$ \\
$\vec{y}_{5}=(-1) \odot \vec{x}_{5}$ & $5.78 \%$ & $14.67 \%$ & $79.56 \%$ \\
$\vec{y}_{6}=(-1) \odot \vec{x}_{6}$ & $6.48 \%$ & $2.22 \%$ & $91.30 \%$ \\
\hline
\end{tabular}

From $\vec{x}_{1}$ to $\vec{x}_{3}$ the results correspond to stand-alone proportional allocation principles and the rest may be understood as proportional allocation principles base on partial contributions. In addition, $\vec{x}_{1}$ and $\vec{x}_{4}$ are linked to GlueVaR ${ }_{99.5 \%, 95 \%}^{11 / 30,2 / 3}, \vec{x}_{2}$ and $\vec{x}_{5}$ to GlueVaR ${ }_{99.5 \%, 95 \%}^{0,1}$ and the remaining were calculated based on GlueVaR $\mathrm{R}_{99.5 \%, 95 \%}^{1 / 20,1 / 8}$. Note that all these relative principles belong to the simplex $\mathscr{S}^{3}$ and have non-zero components.

Assume now that risk managers are interested in allocation principles with a reward to conservative objctives (meaning the smaller the risk figure the better the type of risk). However, the only available information (principles $\vec{x}_{i}, i=1, \ldots, 6$ ) is a set of capital allocation principles with a cost of risk goal. The computation of the inverse of relative capital allocation principles can be useful in this context, in order to obtain principles driven by a reward to a risk minimization objective. So the perturbation inverses of relative capital allocation principles $\vec{x}_{i}, i=1, \ldots, 6$, are shown in Table 8.1 and they are denoted as $\vec{y}_{i}, i=1, \ldots, 6$.

Using the relative principles displayed in (8.10), relative allocation principles are ranked according to Section 8.2.2. As before, symbol $\overrightarrow{0}$ is used to refer to the neutral allocation. The following simplicial distances are calcu- 
lated from expression (8.3):

$$
\begin{aligned}
& \Delta\left(\vec{x}_{1}, \overrightarrow{0}\right)=\sqrt{0.8+0.637+2.865}=2.074, \\
& \Delta\left(\vec{x}_{2}, \overrightarrow{0}\right)=\sqrt{0.911+0.022+1.218}=1.4669, \\
& \Delta\left(\vec{x}_{3}, \overrightarrow{0}\right)=\sqrt{0.453+0.022+0.674}=1.0719, \\
& \Delta\left(\vec{x}_{4}, \overrightarrow{0}\right)=\sqrt{1.083+1.32+4.795}=2.6831, \\
& \Delta\left(\vec{x}_{5}, \overrightarrow{0}\right)=\sqrt{1.404+0.064+2.068}=1.8803, \\
& \Delta\left(\vec{x}_{6}, \overrightarrow{0}\right)=\sqrt{0.276+2.542+4.495}=2.7045 .
\end{aligned}
$$

\section{Figure 8.3 Distances between capital allocation principles.}

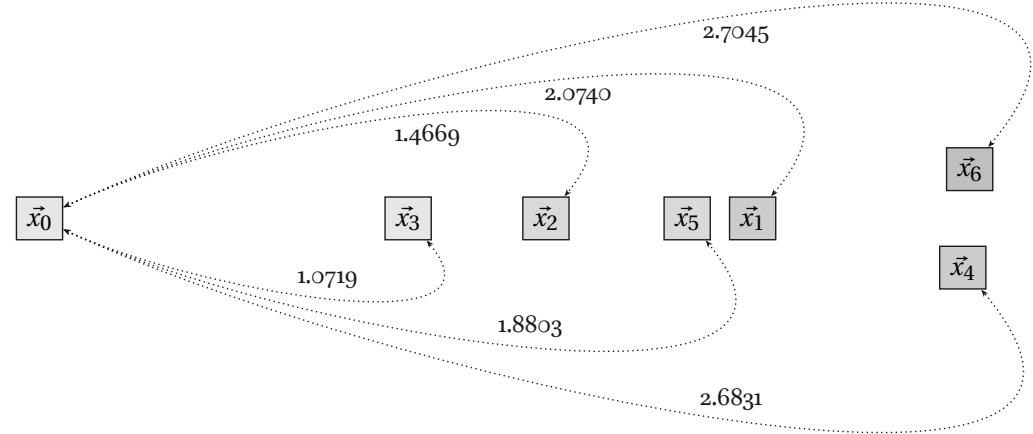

Distances considered individually are not too informative. However, these values allow to rank the principles with respect to one principle of reference, as it is graphically shown in Figure 8.3. From results (8.11), it can be deduced that, in this example, proportional allocation principles based on partial contributions are generally more distant from the neutral allocation than the rest (with $\vec{x}_{1}$ being the only exception). Additionally, when comparing pairs of compositions with the same risk measure involved, it becomes evident that the most different behavior is the one linked to principles depending on GlueVaR $\mathrm{R}_{99.5 \%, 95 \%}^{1 / 20,1 / 8}$ risk measure (the pair $\vec{x}_{3}$ and $\vec{x}_{6}$ ). So, in this example, when using GlueVaR ${ }_{99.5 \%, 95 \%}^{1 / 20,1 / 8}$ as a risk measure, the selection of the allocation criterion (stand-alone versus based on partial contributions) seems to be more relevant than when using the other two GlueVaR risk measures under consideration.

As a final application, the three relative stand-alone allocations displayed in (8.10) are averaged by means of the simplicial arithmetic mean. The rel- 
ative principles based on partial contributions are also averaged. Finally, the simplicial arithmetic mean of these previous averages is obtained, just for the sake of mixing both perspectives in one single principle following a hierarchical approach in which the distribution criterion (as mentioned in Section 6.1 of Chapter 6) plays an important role.

With respect to the stand-alone proportional allocation principles, the geometric means of the three components $(n=3)$ of the three $(m=3)$ relative capital allocations, denoted as $G_{1}, G_{2}$ and $G_{3}$, respectively, are computed. Their values are

$$
\begin{aligned}
& G_{1}=(50.41 \% \cdot 63.51 \% \cdot 54.44)^{1 / 3}=55.86 \%, \\
& G_{2}=(45.8 \% \cdot 28.38 \% \cdot 32.22)^{1 / 3}=34.73 \% \text { and } \\
& G_{3}=(3.79 \% \cdot 8.11 \% \cdot 12.22)^{1 / 3}=7.22 \% .
\end{aligned}
$$

Following expression (8.8), we calculate the value of $\mathscr{C}\left[\left(G_{1}, G_{2}, G_{3}\right)\right]$, i.e. the closure of the vector with components being the geometric means $G_{1}, G_{2}$ and $G_{3}$. By doing so, the value of the simplicial average $\mathrm{AM}_{\Delta}\left(\vec{x}_{1}, \vec{x}_{2}, \vec{x}_{3}\right)$ is obtained which is a relative allocation principle. Similarly, the simplicial arithmetic mean of the relative principles based on partial contributions $\mathrm{AM}_{\Delta}\left(\vec{x}_{4}, \vec{x}_{5}, \vec{x}_{6}\right)$ is also obtained. Both results are shown in Table 8.2.

Table 8.2 Simplicial means of the capital allocation principles

\begin{tabular}{lrrr}
\hline & $X_{1}$ & $X_{2}$ & $X_{3}$ \\
\hline $\mathrm{AM}_{\Delta}\left(\vec{x}_{1}, \vec{x}_{2}, \vec{x}_{3}\right)$ & $57.11 \%$ & $35.51 \%$ & $7.38 \%$ \\
$\mathrm{AM}_{\Delta}\left(\vec{x}_{4}, \vec{x}_{5}, \vec{x}_{6}\right)$ & $46.64 \%$ & $50.60 \%$ & $2.74 \%$ \\
\hline
\end{tabular}

As it can be proved, the components of the simplicial averages are not equal to the arithmetic mean of the components of the original principles. In fact, the components of the simplicial average are linked to the geometric mean of the components of the original relative principles. As a final result, let us average the two principles displayed in Table 8.2. In this case, the geometric means of the three components $(n=3)$ of the two $(m=2)$ relative capital allocations, denoted as $G_{1}^{\prime}, G_{2}^{\prime}$ and $G_{3}^{\prime}$, respectively, are computed. Their values are

$$
G_{1}^{\prime}=\sqrt{57.11 \% \cdot 46.64 \%}=51.61 \%,
$$




$$
\begin{aligned}
G_{2}^{\prime} & =\sqrt{35.51 \% \cdot 50.62 \%}=42.39 \% \text { and } \\
G_{3}^{\prime} & =\sqrt{7.38 \% \cdot 2.74 \%}=4.5 \% .
\end{aligned}
$$

In order to obtain the final result, we need to calculate the closure of $\vec{w}=$ $\left(G_{1}^{\prime}, G_{2}^{\prime}, G_{3}^{\prime}\right)$. This is

$$
\mathrm{AM}_{\Delta}\left(\mathrm{AM}_{\Delta}\left(\vec{x}_{1}, \vec{x}_{2}, \vec{x}_{3}\right), \mathrm{AM}_{\Delta}\left(\vec{x}_{4}, \vec{x}_{5}, \vec{x}_{6}\right)\right)=\mathscr{C}[\vec{w}]
$$

This relative principle is

$$
\text { (52.40\%, 43.04\%, 4.57\%). }
$$

Final remark. Another feasible approach to reach this unique allocation would be to calculate the simplicial arithmetic mean of the whole set of relative principles shown in (8.10). It has to be noted that the result would certainly be different, because this last approach would lack the specific aggregation hierarchy that we have imposed herein.

\subsection{Exercises}

1. In the example presented in Section 8.2.4, calculate the simplicial distance between $\mathrm{AM}_{\Delta}\left(\mathrm{AM}_{\Delta}\left(\vec{x}_{1}, \vec{x}_{2}, \vec{x}_{3}\right), \mathrm{AM}_{\Delta}\left(\vec{x}_{4}, \vec{x}_{5}, \vec{x}_{6}\right)\right)$ and $\mathrm{AM}_{\Delta}\left(\vec{x}_{1}\right.$, $\vec{x}_{2}, \vec{x}_{3}, \vec{x}_{4}, \vec{x}_{5}, \vec{x}_{6}$ ) where $\vec{x}_{i}, i=1, \ldots, 6$, are the relative principles shown in (8.10).

2. Observe the distances between principles shown in the example presented in Section 8.2.4, find a stand-alone allocation which is located between $\vec{x}_{1}$ and $\vec{x}_{2}$.

3. Assume an allocation principle in a situation of $n$ different sources that assigns an allocation equal to $2 i /(n(n+1))$ for the risk $i, i=1, \ldots, n$. Show that this is not the neutral allocation and find the distance to this element as a function of $n$. Calculate the behaviour of this distance as $n$ increases.

4. In the same situation as in the previous exercise, propose another allocation for $i, i=1, \ldots, n$, different to the neutral allocation and compare it to $2 i /(n(n+1))$ for the risk $i, i=1, \ldots, n$.

5. Assume a situation of $n$ different sources that assigns an allocation equal to $2 i /(n(n+1))$ for the risk $i, i=1, \ldots, n$, find an average allocation that 
summarizes the allocations provided by this one and the neutral allocation. Find the average allocation now also with the third allocation that you proposed in the previous exercise. 